\title{
Contemporary Interior Design in Low-Carbon Context Lijuan
}

\author{
Zhang \\ Zaozhuang University, Zaozhuang, Shandong, 277160 \\ 290882312@qq.com
}

\section{Keywords: Low-carbon; Interior design; Reflection; Development}

\begin{abstract}
Since the middle and late $20^{\text {th }}$ century, environmental issues have become increasingly prominent. "Low carbon" has become an important context for current economic and social development. Therefore, the low-carbon concept must be introduced into interior design to alleviate the current environmental crisis while fully satisfying people's physiological, psychological and spiritual needs and creating an ideal living space, in order to create a harmonious and comfortable, low-carbon awareness naturalized and humanized interior space.
\end{abstract}

\section{Introduction}

Since the middle and late $20^{\text {th }}$ century, the environmental crisis has become increasingly prominent and has become the focus of attention. Serious environmental problems do not only hinder the development of economic and social undertakings, but also threaten the survival of people. Under this background, the concept of low-carbon began to rise and gradually extended to economic, social, cultural and daily life. According to relevant statistics, nearly $60 \%$ of the world's timber and $50 \%$ of the world's water resources are used in the construction industry. The construction industry has an important influence on solving environmental problems. As a related industry in the construction industry, interior design is also a high-energy-consuming industry and is closely related to architecture, society, folk customs, interior furnishings, decorative materials and performance. Therefore, we must introduce low-carbon concepts into interior design, pay attention to the scientificity and standardization of the design process, highlight the concept of sustainable development of modern design, and create a natural and humanized interior space that reflects harmony and comfort and reflects low-carbon awareness.

\section{Reflections on Current Interior Design in Low Carbon Environment}

In recent years, with the continued construction of urbanization in China, the interior design industry has also been booming. However, we should see that in the current low-carbon context, many problems have arisen in the development process of interior design, causing great damage to the ecological environment and adversely affecting people's daily lives, which is mainly shown in the following areas:

First of all, with the rapid development of China's economic and social undertakings, people are no longer satisfied with the basic needs of material life, and began to pursue spiritual enjoyment, optimizing and beautifying their living environment became an important part of daily life. At present, some interior designs pay attention to and cater to this trend in real life and regard interior design as an important way to satisfy people's vanity and luxury. Therefore, there are a large number of design works that pursue novelty, fashion and style.

Second, the decorative materials used in interior design can harm the human body, not only polluting the environment or damaging the indoor air, but also damaging people's health. For example, many decorative materials used in current interior design contain volatile organic compounds, such as formaldehyde and benzene. Stay in such an environment for a long time, people's physical and mental health will be faced with great harm.

Third, the emphasis on innovation of interior design has caused a great deal of waste of resources. In recent years, due to the ever-changing interior design style, it has contributed to the tendency of people to change their decoration style at any time according to their preferences. This has led to a 
large amount of waste in the design and decoration process and increased energy consumption.

In the end, there is a tendency to ignore functions, pursuing the decorative form of interior design and ignoring the practical needs of everyday life. At present, many interior designs have one-sided emphasis on the artistry of design and neglect the satisfaction of the actual functions of the living room, which does not only result in the waste of a large amount of decorative materials and resources, but also reduces people's quality of life.

It can be seen that there are many outstanding problems in the current interior design in the low-carbon context. To solve these problems, it is necessary to carry out innovative research on interior design, actively promote the transformation of interior design, and make the interior design take the road of sustainable development.

\section{Low Carbon Concept Promotes the Transformation of Contemporary Interior Design}

With environmental issues becoming increasingly prominent, the low-carbon concept has attracted the attention of the society and gradually penetrated into the fields of economy, culture and life. Under the influence of the low-carbon concept, interior design should fully meet people's physical, psychological and spiritual needs while alleviating the current shortage of resources in environmental crisis, and create an ideal living space. Therefore, we must actively respond to the appeal of low-carbon concepts and promote the transformation and development of contemporary interior design.

Pay Attention to the Optimization of Interior Space Environment. In a sense, the interior design is the transformation of the living room building space. To a certain extent, it re-processes the interior environment scale and the proportional relationship, and merges or connects some functions of the living room, excluding the strict limitation of the interior space of the building itself. By appropriately changing the spatial shape, position or size of the interior of the living room, the function of the living room can be further optimized, which cannot only satisfy people's daily needs, but also can show strong adaptability and flexibility.

Emphasize the Practical Features of the Decoration Design. Interior design is mainly based on certain design requirements, optimizes and deals with the interior space of the building to fully meet people's real life needs. For example, in the process of designing and decorating, consciously choosing to use low-carbon and environmentally-friendly decorative materials cannot only create a unique living environment and artistic atmosphere, satisfy people's spiritual needs, but also can provide functions, such as heat insulation, moisture-proof, waterproof, and sound insulation, to extend the service life of residential space and improve people's daily life quality.

Highlight the Importance of Soft Furnishings. Contemporary interior design emphasizes the low-carbonization of interior furnishings and creates a unique living environment through a rational selection of furniture, comprehensive treatment of decorative fabrics, lighting and indoor planting. For example, under the influence of the concept of low-carbon life, people pursue a natural lifestyle. Therefore, the contemporary interior design should pay attention to the application of natural materials, use the folk art techniques and styles to create new texture effects and a unique living space.

\section{The Strategy of Contemporary Interior Design in Low-Carbon Context}

In the face of today's increasingly serious environmental problems and survival crisis, people gradually establish a low-carbon life concept, actively promote the harmony between man and nature, and promote the coordinated development of social development and ecological protection. As an important industry which is closely related to the daily life of contemporary people, interior design should strive to reduce the resource consumption during the decoration design process, explore and establish a sound interior design thinking model, and create a more energy-efficient, environmentally friendly and more humane living environment, to meet people's real-life needs and spiritual and cultural needs. 
In the Function of the Living Room, Pursue Comfort of Living. Under the influence of the low-carbon concept, contemporary interior design should adopt low-carbon environmental protection technologies and ecological energy conservation methods to fully meet people's needs for interior functions and improve people's daily living comfort. First of all, from the people's daily needs and the physiological characteristics of the user, the architectural space should be appropriately reconstructed and rationally optimized, and the tendencies of unilaterally pursuing large space and large scale in the process of interior design should be eliminated. Second, on the basis of the full use of the original indoor space layout, lighting, ventilation and energy conservation, according to the needs of people's daily lives, we will flexibly use certain technical means to transform and optimize and to create a natural, simple and friendly living environment. Finally, according to the concept of low-carbon life, practice a healthy, green and environmentally friendly lifestyle, try to use local architectural decoration materials to create a natural and comfortable living environment through ingenious design and reasonable planning.

In Interior Design, Emphasis on the Natural Nature of Design Style. The fundamental purpose of the low-carbon concept is to promote harmony between man and nature and to safeguard human survival and development. Therefore, interior design should fully support the goal of human physical health, actively explore the aesthetics of interior design, and achieve the naturalness of interior design style. First of all, we should give full play to the role of decorative design techniques, such as modeling, greening, and furnishings in interior design, and further beautify people's visual psychological space. Secondly, appropriately use eco-environmental technologies, such as solar energy and wind energy, to save water, electricity and other resources needed by people in daily life, effectively reducing energy consumption. Finally, the interior design should optimize the interior space through concise artistic modeling, improve the visual space structure and create an interior environment with humanistic and natural features.

In Terms of Material Use, Importance is Placed on Material Safety. Interior design cannot be separated from the use of materials. In the low-carbon context, contemporary interior design should choose low-energy, low-pollution and low-cost building decoration materials to improve people's quality of life. On the one hand, in the process of design and decoration, renewable and pollution-free environmentally friendly materials are used to give full play to the advantages of renewable and degradable synthetic materials, such as steel, glass and PVC, and to prevent the selection of "wood, bamboo, leather, rattan" and other materials which will cause environmental damage and waste of resources. The comfort and "humanization" of daily life should be based on the harmony between human and natural ecology. On the other hand, in the process of interior design, the concept of "paying more attention on design, not decoration" is established to minimize the use of decorative materials and to prevent the release of toxic and harmful gases during using these materimals which will cause damage to interior environment and reduce the quality of life of people.

In Terms of Design Style, Highlight the Traditional Character. In the process of designing and decorating, interior design should actively seek the resources of Chinese national traditional culture based on the reasonable experience of absorbing Western architectural interior design, and actively explore the design style and methods with distinctive local characteristics. First of all, we must fully study and carry forward the traditional Chinese concepts of interior decoration, and draw inspiration and wisdom from the excellent decorative buildings in ancient China, which are the ideas of "harmony between man and nature" and "co-existence", which have "low carbon environmental protection" and other features. Secondly, on the basis of exploring the style of design works, we must give full play to the practical functions of interior space, extend the service life of interior design and decoration projects, and increase the practical value of interior design. Finally, the essential attributes of contemporary interior design are clearly defined, and the factors, such as space, form, material, color, lighting and furnishings, as well as the development trend of the field are clearly grasped. The interior space which meets the daily needs of people with the traditional ethnic characters and the modern humanistic moods. 


\section{Conclusion}

In the face of the increasingly serious environmental crisis and the development requirements of low-carbon economy, interior design should firmly establish the concept of low-carbon life. In the design process, natural environment factors must be fully taken into consideration, and the role of ecological technologies, materials and techniques should be effectively used to actively explore the unity, coordination and interactive development between the functional comfort of the interior design and the beautiful environment under the concept of low carbon and environmental protection, in order to create a unique environment with reasonable functions, comfortable and beautiful, and satisfy people's material and spiritual life needs, so as to meet people's material and spiritual needs in a low-carbon environment.

\section{References}

[1] Wei Shan. Ecological Design Concept in Interior Furnishing Art [J]. Art Observation, 2009, (5).

[2] Yuming Du. The Embodiment of Low-carbon Economy in Interior Design[J]. Theory of Literature and Art, 2010, (12).

[3] Yuanbo Zhong. Thoughts on Ecological Interior Design[J]. Decoration, 2005, (10).

\footnotetext{
About the author: Lijuan Zhang (1983-), female, Jining, Shandong; Master; Lecturer, Research direction: interior design.

Address: Art and Design School, Zaozhuang College, No. 1, Beian Road, Shizhong District, Zaozhuang City, Shandong, 277160
} 